\title{
Upregulation and activation of p53 by erastin-induced reactive oxygen species contribute to cytotoxic and cytostatic effects in A549 lung cancer cells
}

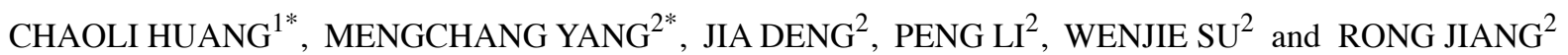 \\ ${ }^{1}$ Department of Nephrology, Affiliated Hospital, Chengdu University, Chengdu, Sichuan 610081; \\ ${ }^{2}$ Department of Anesthesiology, Sichuan Academy of Medical Sciences and Sichuan Provincial People's Hospital, \\ Chengdu, Sichuan 610072, P.R. China
}

Received December 5, 2017; Accepted June 21, 2018

DOI: $10.3892 /$ or.2018.6585

\begin{abstract}
The tumour-suppressor protein p53 is a key regulator of multiple cellular processes and exerts its tumour-suppressor function by inducing apoptotic cell death. However, emerging evidence indicates that $\mathrm{p} 53$ is also involved in inducing ferroptosis, which is a unique iron-dependent form of non-apoptotic cell death triggered by the RAS-selective lethal small molecule erastin. Previous studies have shown that erastin exposure induces increased ROS accumulation and oxidative stress. In the present study, we incubated A549 cells with erastin and detected ROS accumulation. Semi-quantitative western blotting was performed to analyse the effect of the induced ROS on p53 activity. To determine how ROS activate p53, NAC, an ROS scavenger, and KU-55933, an ATM kinase inhibitor, were employed to co-incubate with erastin, followed by western blot analysis. Either p53 or SLC7A11 siRNA was introduced into A549 cells to silence the target-gene expression, followed by ROS detection to illustrate the regulatory role of ROS-activated p53 on its target gene SLC7A11. Annexin V-FITC/PI staining was performed to detect the induction of apoptotic cell death by erastin exposure. To further assess the effects of erastin treatment on cellular proliferation, EdU staining and cell cycle flow cytometric analysis were performed. Erastin exposure upregulated and activated p53 and thus, transcriptionally activated its downstream target genes, including p21 and Bax, in lung cancer A549 cells dependent on erastin-induced ROS. Subsequently, activated p53 by erastin treatment suppressed SLC7A11 and induced ROS accumulation, indicating the
\end{abstract}

Correspondence to: Professor Rong Jiang, Department of Anesthesiology, Sichuan Academy of Medical Sciences and Sichuan Provincial People's Hospital, 32 Wester Second Section First Ring Road, Chengdu, Sichuan 610072, P.R. China

E-mail: 1076819702@qq.com

${ }^{*}$ Contributed equally

Key words: p53, apoptosis, ferroptosis, ROS, erastin, SCL7A11 potential feedback loop between p53 and erastin-induced ROS. By employing the caspase inhibitor Z-VAD-FMK, it was revealed that erastin-induced p53 contributed to both ferroptotic and apoptotic cell death and inhibited cell proliferation via arresting the cell cycle at G1 phase. Collectively, these results indicated that p53 may contribute to the cytotoxic and cytostatic effects associated with establishing a feedback loop with ROS induced by erastin.

\section{Introduction}

Erastin is a voltage-dependent anion channel (VDAC)-binding small molecule and exerts cytotoxic effects on several selective cancer cells $(1,2)$. Erastin exposure induces the accumulation of reactive oxygen species (ROS) in an iron-dependent manner. The direct binding of erastin to voltage-dependent anion-selective channel protein 2 (VDAC2) and 3 (VDAC3) is necessary to induce ferroptosis, which involves a unique constellation of morphological, biochemical and genetic features and is distinct from apoptosis, various forms of necrosis and autophagy (3). Erastin also exerts cytotoxic effects on several human cancer cell lines by inducing oxidative stress and caspase-9-dependent apoptotic death (4), indicating that erastin potentially induces ferroptotic and apoptotic cell death.

Being the most well-known tumour suppressor, p53 exerts multi-functional roles in controlling cell cycle checkpoints, apoptosis and DNA repair (5). In addition to these commonly accepted functions mediated by activated p53, accumulating evidence indicates that other activities of p53 are also involved in tumour suppression, including ferroptosis. It was reported that p53 post-transcriptionally inhibited the expression of SLC7A11, a key component of the cysteine/glutamate antiporter, leading to inhibition of cysteine uptake and sensitization of cells to ferroptosis (6). The suppression of SLC7A11 by p53 subsequently leads to the reduction of glutathione production and ROS accumulation, which are important components of ferroptosis (6). Gao et al identified GLS2, a p53-regulated glutaminase, as essential for ferroptosis (7). All of these studies support the potential relevance of p53 to ferroptosis and the tumour-suppressing activity of p53 via the regulation of ferroptosis. Thus, we investigated the potential 
mechanism underlying how p53 participates in the regulation of erastin-induced ferroptosis.

Erastin-induced ferroptotic cell death is dependent on the increased level of intracellular reactive oxygen species (ROS), which are widely believed to act as a mediator of apoptosis. Tsai et al (8) found that, in A549 non-small cell lung cancer cells, the activation of p53-dependent apoptotic proteins, including PUMA, cytochrome $c$, Apaf-1 and caspase-3, was dependent on the presence of ROS. Accumulated ROS were also reported to regulate the expression and activation of $\mathrm{p} 53$, and the ROS/p53 pathway was found to regulate several cellular physiological processes, including cell senescence (9), oxidative protection (10) and apoptosis (11). In erastin-induced ferroptotic death, p53 was activated as a post-transcriptional suppressor of SLC7A11 and induced ROS generation (6). By considering that DNA damage induced by accumulated ROS activates p53 (8), the question was raised whether induction of ROS by erastin exposure was attributed to p53 activity.

In the present study, we investigated the regulatory effects of ROS induced by erastin exposure on p53 expression in A549 cells, and we studied the effects of the activation of p53 on cell proliferation and apoptosis. In the present study, we revealed that the markedly increased expression of p53 in A549 cells following erastin exposure was partially dependent on the accumulation of ROS. We concluded that the ROS/p53 pathway activated by erastin exposure exerted cytotoxic and cytostatic effects on A549 cells via both ferroptosis and apoptosis.

\section{Materials and methods}

Cell culture and treatment. The human adenocarcinoma A549 and lung fibroblast WI-38 cell lines were purchased from the American Type Culture Collection (ATCC; Manassas, VA, USA) and maintained in Gibco ${ }^{\mathrm{TM}}$ Dulbecco's modified Eagle's medium (DMEM; Thermo Fisher Scientific, Inc., Paisley, UK) supplemented with $10 \%$ fetal bovine serum (FBS), $100 \mathrm{U} / \mathrm{ml}$ of penicillin and $0.1 \mathrm{mg} / \mathrm{ml}$ of streptomycin (all from Thermo Fisher Scientific, Inc.). For different analyzing purposes, A549 cells was treated with $3.12 \mu \mathrm{M}$ erastin (Sigma-Aldrich, St. Louis, MO, USA) for $24 \mathrm{~h}$, NAC (Sigma-Aldrich) $5 \mathrm{mM}$ for $4 \mathrm{~h}$ or KU-55933 (Selleck Chemicals, Shanghai, China) $15 \mathrm{nM}$ for $24 \mathrm{~h}$. Then cells were harvested as described below for further analysis.

Western blotting. Cells were harvested and pelleted by centrifugation at $1,000 \times \mathrm{g}$ and $4^{\circ} \mathrm{C}$ for $10 \mathrm{~min}$ and washed with ice-cold PBS twice. Lysis buffer $(100 \mu \mathrm{l})$ containing $50 \mathrm{mM}$ Tris- $\mathrm{HCl}, 150 \mathrm{mM} \mathrm{NaCl}, 0.02 \% \mathrm{NaN}_{3}, 100 \mu \mathrm{g} / \mathrm{ml}$ phenylmethanesulfonyl fluoride (PMSF), $1 \mu \mathrm{g} / \mathrm{ml}$ aprotinin, $1 \mu \mathrm{g} / \mathrm{ml}$ pepstatin $\mathrm{A}$, and $1 \%$ Triton $\mathrm{X}-100$ was each added into $1 \times 10^{6}$ cells for obtaining the cell lysate. After centrifugation at $12,000 \mathrm{x} \mathrm{g}$ for $10 \mathrm{~min}$ at $4^{\circ} \mathrm{C}$, the supernatant was collected and assessment of protein concentration was carried out using a bicinchoninic acid (BCA) protein assay kit (Sigma-Aldrich; Merck KGaA, Darmstadt, Germany). Protein (50 $\mu \mathrm{g}$ ) was resolved by $10 \%$ sodium dodecyl sulfate-polyacrylamide gel electrophoresis (SDS-PAGE) and then transferred to a nitrocellulose membrane. After transferring, the membrane was blocked using $5 \%$ bovine serum albumin (BSA) in PBS for $30 \mathrm{~min}$ at room temperature. The primary antibodies against p53 (1:5,000 dilution; cat. no. ab28), phosphorylated p53 (1:2,000 dilution; cat. no. ab1431), p21 (1:1,000 dilution; cat. no. ab109520), Bax (1:2,000 dilution; cat. no. ab32503), SLC7A11 (1:1,000 dilution; cat. no. ab37185) and $\beta$-actin (1:5,000 dilution; cat. no. ab8226) were purchased from Abcam (Cambridge, UK) and incubated with the blocked membrane separately for $2 \mathrm{~h}$ at room temperature. After being washed 3 times for 5 min each with PBS-T, the membrane was incubated for $1 \mathrm{~h}$ with peroxidase-coupled secondary antibodies (1:5,000 dilution; cat. nos. ab6785 or ab6721), and detected with the ECL Plus Western Blotting Detection reagents (Pierce Biotechnology, Rockford, IL, USA) and imaged using X-ray film.

CFSE/PI double staining. A549 cells were washed in phosphate-buffered saline (PBS) twice and $100 \mu \mathrm{l}$ of CFSE fluorescent dye $(50 \mu \mathrm{mol} / \mathrm{l}$; Thermo Fisher Scientific, Inc., Waltham, MA, USA) was added, followed by incubation at $37^{\circ} \mathrm{C}$ for $30 \mathrm{~min}$. Cells were washed in PBS twice and incubated in medium supplemented with $10 \% \mathrm{FBS}$ for another 24-h incubation. After two washes with PBS, PI $(20 \mu \mathrm{g} / \mathrm{ml}$; Sigma-Aldrich) was added for incubation at room temperature for $10 \mathrm{~min}$. After two washes with PBS, cells were imaged under a X71 (U-RFL-T) fluorescence microscope (Olympus, Melville, NY, USA).

EdU staining. Cells were plated in 12-well plate and allowed to attach overnight. In addition, 5-ethyny-2'-deoxyuridine (EdU) (Cell-Light EdU Cell Proliferation Detection kit; Guangzhou RiboBio Co., Ltd., Guangzhou, China) was used as a marker of cell proliferation. EdU was added at a final concentration of $50 \mu \mathrm{mol} / 1$ into the medium and the cells were cultured for an additional $120 \mathrm{~min}$. Cells were washed twice with PBS and fixed with $4 \%$ paraformaldehyde at room temperature for $10 \mathrm{~min}$, washed with glycine $(2 \mathrm{mg} / \mathrm{ml})$ for $5 \mathrm{~min}$ in a shaker, treated with $0.2 \%$ Triton $\mathrm{X}-100$ for $10 \mathrm{~min}$ and washed with PBS twice. Click-iT ${ }^{\circledR}$ Cell Reaction Buffer kit (Thermo Fisher Scientific, Inc.) was added for further incubation for $30 \mathrm{~min}$. Then the cells were washed with $0.5 \%$ Triton X-100 for three times, stained with DAPI for $10 \mathrm{~min}$ at room temperature, washed with $0.5 \%$ Triton X-100 for three times, immersed in $150 \mu \mathrm{l}$ of PBS and examined under a X71 (U-RFL-T) fluorescence microscope (Olympus).

Cell viability assay. A549 cells were suspended and adjusted to $1 \times 10^{6}$ cells $/ \mathrm{ml}$ and 5,000 cells/well were plated into a 96-well plate and incubated overnight. Various concentrations of erastin $(0.1,1,2,4,6,8$ and $10 \mu \mathrm{M})$ were added into each well. Twenty-four hours later, the Cell Counting Kit-8 (CCK-8, Sigma-Aldrich; Merck KGaA, Darmstadt, Germany) prepared solution was added for a 4 -h co-incubation at $37^{\circ} \mathrm{C}$ in the dark. Absorbance at wavelength $450 \mathrm{~nm}$ (A450) was detected by a microplate reader (Synergy 2 Multi-Mode Microplate Reader; BioTek, Winooski, VT, USA) to determine the cell viability.

Assessment of ROS. Cells were co-incubated with [5-(and 6)-carboxy-2',7'-dichlorodihydrofluorescein diacetate] (carboxy- $\mathrm{H}_{2}$ DCFDA; Thermo Fisher Scientific, Inc.) at a 
final concentration of $5 \mu \mathrm{mol} / 1$ for $30 \mathrm{~min}$. Subsequently the medium was removed and washed twice with PBS. The green fluorescence was imaged using a X71 (U-RFL-T) fluorescence microscope (Olympus). For quantitative measurement, stained cells were suspended using $0.25 \%$ trypsin (Gibco; Thermo Fisher Scientific, Inc.) and the green fluorescence was assessed using a 3-laser Navios flow cytometer (Beckman Coulter Inc., Brea, CA, USA).

$R N A$ interference. Invitrogen ${ }^{\mathrm{TM}}$ Oligofectamine transfection reagent (Thermo Fisher Scientific,Inc.) was used for transfecting $50 \mathrm{nM}$ p53 siRNA (p53si), SLC7A11 siRNA (SLC7A11si) or negative control siRNA (CTLsi) purchased from Ambion (Life Technologies; Thermo Fischer Scientific, Inc.) into A549 cells according to the manufacturer's instructions and then after $24 \mathrm{~h}$, the cells were used for subsequent experiments.

Immunofluorescence assay. Cells were washed with ice-cold PBS for 5 min, 3 times and fixed with ice-cold alcohol for $15 \mathrm{~min}$ at $4^{\circ} \mathrm{C}$. Subsequently, the cells were blocked with $1 \%$ BSA, $0.1 \%$ Triton X-100 in PBS for $1 \mathrm{~h}$ at room temperature. Then the cells were incubated with anti- $\gamma \mathrm{H} 2 \mathrm{AX}$ antibody (cat. no. ab26350; Abcam) at a 1:200 dilution for $2 \mathrm{~h}$ at room temperature. After 3 washes with ice-cold PBS, the cells were incubated with Alexa Fluor ${ }^{\circledR}$ 647-conjugated donkey anti-mouse IgG secondary antibody (cat. no. ab150107; Abcam) at a 1:2,000 dilution for $1 \mathrm{~h}$ at room temperature. After 3 washes with ice-cold PBS, the cells were incubated with $5 \mu \mathrm{g} / \mathrm{ml}$ DAPI for $10 \mathrm{~min}$. The slices were analyzed under a X71 (U-RFL-T) fluorescence microscope (Olympus) at a magnification of $\mathrm{x} 200$.

Cell cycle analysis. Cells $\left(1 \times 10^{6}\right)$ were collected and fixed in $70 \%$ ice-cold ethanol at $-20^{\circ} \mathrm{C}$ overnight. Then the cells were collected and resuspended in PBS supplemented with $100 \mathrm{ng} / \mathrm{ml}$ RNase A and $50 \mathrm{ng} / \mathrm{ml}$ propidium iodide (PI) for $30 \mathrm{~min}$. After staining, samples were analyzed for cell cycle distribution with a 3-laser Navios flow cytometer (Beckman Coulter). Experiments with duplicates were performed independently thrice.

Assessment of caspase-3 activity. A caspase-3 colorimetric assay kit (R\&D Systems, Minneapolis, MN, USA) was used to detect the enzyme activity of caspase-3. The total cell lysate was qualified by the BCA protein assay kit (Sigma-Aldrich; Merck KGaA). Total protein $(50 \mu \mathrm{g})$ for each sample was moved to a 96-well microplate and quantified using a microplate reader (Synergy 2 Multi-Mode Microplate Reader; BioTek).

Cell apoptosis. Cells $\left(5 \times 10^{5}\right)$ were collected and co-incubated with $5 \mu$ l Annexin V-fluorescein isothiocyanate (Annexin V-FITC) and $10 \mu \mathrm{l}$ PI supplied by an Annexin V/PI apoptosis kit (BioVision, San Francisco, CA, USA) for $10 \mathrm{~min}$ in the dark. After staining, the ells were subjected to flow cytometric analysis (3-laser Navios flow cytometer; Beckman Coulter).

Statistical analysis. All data are presented as the mean \pm SD. Statistical differences among different groups were analyzed by one way analysis of variance (ANOVA) with Dunnett's post hoc test using Prism 6 (GraphPad Software, Inc., San Diego, CA, USA). A value of $\mathrm{P}<0.05$ or $\mathrm{P}<0.01$ was considered to indicate a statistically significant difference.

\section{Results}

ROS upregulate and activate p53 in response to erastin exposure. To ascertain whether erastin exerts a cytotoxic effect and increases ROS in A549 cells and the non-tumour cell line WI-38, both cell types were treated with increasing concentrations of erastin $(0.1-10 \mu \mathrm{M})$ and were subjected to the CCK-8 assay $24 \mathrm{~h}$ later to assess cell viability. As displayed in Fig. 1A, exposure to $0-10 \mu \mathrm{M}$ erastin for $24 \mathrm{~h}$ significantly decreased A549 cell viability in the A549 cells, but not that of WI-38 cells. Erastin has been reported to play a critical role in inducing ferroptosis via ROS accumulation (3). We quantified fluorescence in erastin-treated $\left(\mathrm{IC}_{30}\right.$ concentration for A549 cells, $3.12 \mu \mathrm{M}$ ) A549 or WI-38 cells stained with a redox-sensitive probe. Consistent with previous literature (6), erastin treatment increased the ROS level in A549 cells but not that in WI-38 cells (Fig. 1B), indicating that NSCLC A549 cells are much more sensitive than non-tumour WI-38 lung cells. Thus, subsequent experiments were focused on the effects of erastin treatment on A549 cells. To determine the role of ROS induced by erastin exposure on $\mathrm{p} 53$, the expression levels of p53 protein and its transcriptional targets Bax and p21 were detected. As displayed in Fig. 1C, the upregulation and activation of p53 were evidenced by upregulated p53, Bax and p21. Following pretreatment with $N$-acetyl-1-cysteine (NAC), an ROS scavenger, erastin exposure failed to significantly affect p53 activation, indicating that activation was dependent on the presence of ROS induced by erastin exposure (Fig. 1C, right panel). According to the literature, activated p53 transcriptionally suppresses SLC7A11, a key component of the cysteine/glutamate antiporter, thus promoting ferroptosis induced by erastin exposure (12). This prompted us to detect the expression of SLC7A11 in A549 cells with or without p53 knockdown after erastin exposure. In the CTLsi group, with the upregulation of p53 induced by erastin treatment, SLC7A11 was obviously downregulated (Fig. 1D). While there was no obvious effect of erastin treatment on the expression of SLC7A11 in A549 cells with p53 knockdown, progressive suppression of the SLC7A11 protein level was observed in A549 cells exposed to erastin (Fig. 1D). These results indicated that ROS induced by erastin potentially contributed to ferroptosis induction via activating p53 transcriptional activity.

Erastin-induced ROS lead to the DNA damage response and stimulate p53 in A549 cells. By considering the effect of accumulated ROS on the DNA damage response (DDR) $(13,14)$, we then tested whether erastin-induced ROS caused DDR in A549 cells. As expected, erastin exposure produced some typical $\gamma$-H2AX positive-stained cells. Conversely, co-exposure of erastin with NAC only produced few $\gamma$-H2AX positive-stained cells (Fig. 2A). Without disturbing p53 mRNA levels (data not shown), the activation of p53 by erastin was abolished by NAC co-incubation (Fig. 2B). DDR is responsible for inducing p53 post-transcriptionally, which is phosphorylated by activated ataxia telangiectasia mutated (ATM) kinase (15), and prompted 

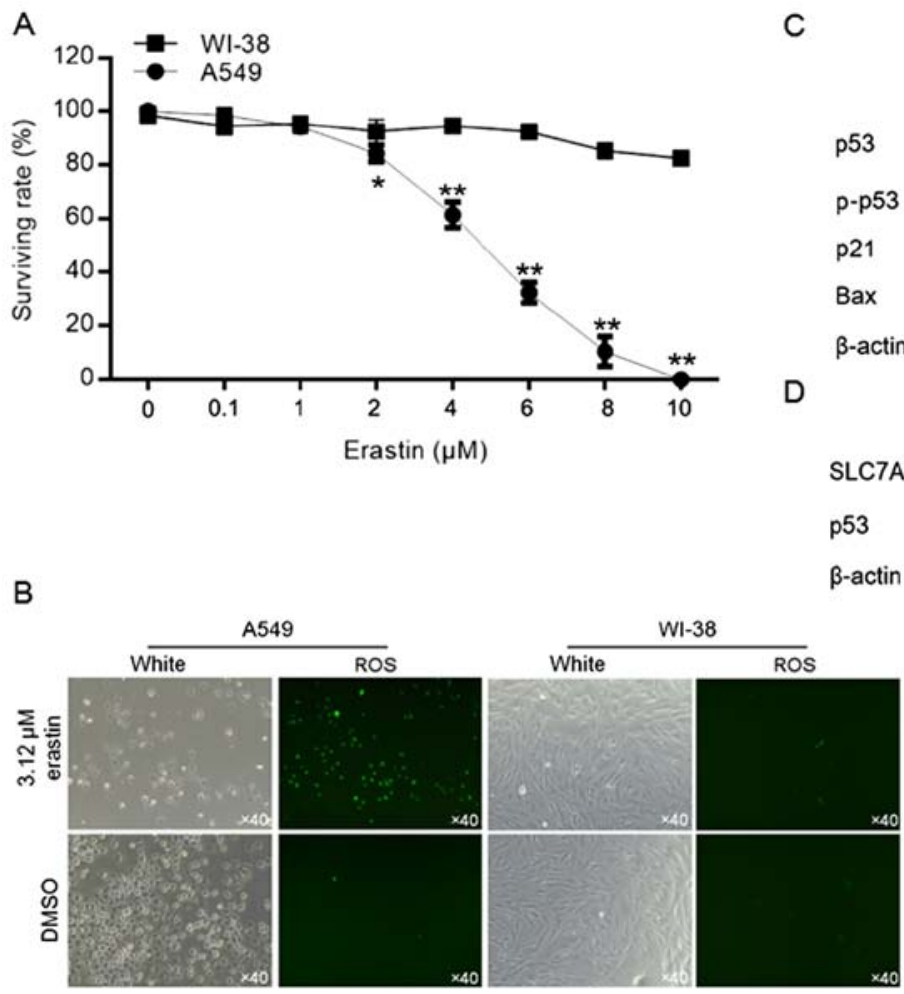

C

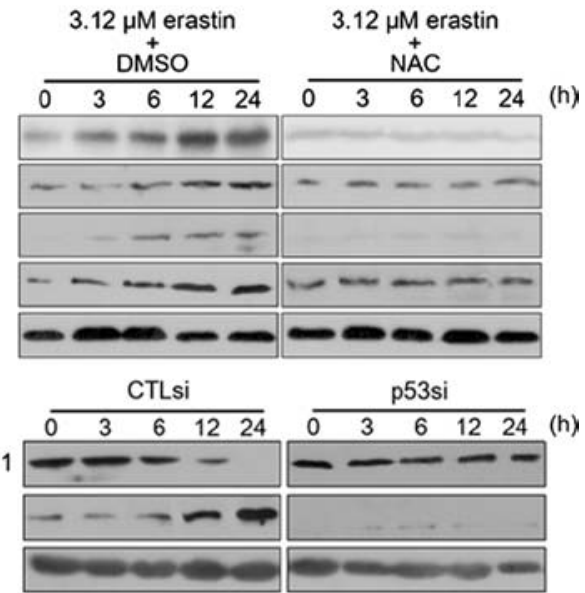

Figure 1. ROS induced by erastin exposure upregulate and activate p53 in A549 cells. (A) Erastin exposure presented cytotoxicity to A549 cells, but not to WI-38 cells. (B) Erastin exposure induced ROS generation in A549 cells, but not in WI-38 cells. (C) Erastin exposure upregulated and activated p53 dependent on the accumulation of ROS in A549 cells. (D) Upregulated p53 by erastin exposure suppressed the expression of SLC7A11 in A549 cells. "P<0.05; ${ }^{* *} \mathrm{P}<0.01$ vs. the DMSO group.

us to determine whether ROS induction of p53 is dependent on DDR and subsequent activation of phosphorylation. Thus, KU-55933, a specific ATM inhibitor, was employed to inhibit the phosphorylation of $\mathrm{p} 53$ at S15 by DDR-activated ATM, and then the effects of accumulated ROS on p53 were detected. As displayed in Fig. 2C and D, NAC treatment, but not KU-55933, induced DDR, and both NAC and KU-55933 treatment clearly inhibited p53 phosphorylation following erastin exposure, indicating that the activating effects of erastin-induced ROS on p53 were exerted via ATM kinetic activity after causing DDR (Fig. 2E).

Expression of p53 increases erastin-induced ROS generation partially dependent on decreasing the expression of SCL7A11. To determine the role of activated p53 on ROS generation induced by erastin, we assessed the ROS level in A549 cells with or without p53 knockdown in A549 cells after erastin exposure. As illustrated in Fig. 3A, while erastin exposure induced accumulating ROS in both A549-p53si and A549-CTLsi cells, the ROS level in A549-p53si cells was lower than that in A549-CTLsi cells. According to research, activated p53 suppresses post-transcriptionally the expression of SLC7A11, a key component of the cysteine/glutamate antiporter, thus inducing ROS accumulation (12). This prompted us to ascertain whether ROS induction by activated p53 after erastin exposure was dependent on the suppression of SLC7A11. We employed SCL7A11si to efficiently knock down the expression of SCL7A11 without disturbing the expression of p53 (Fig. 3B). After erastin exposure, both A549-SLC7A11si and A549-SLC7A11si/p53si cells presented decreased ROS levels. By comparison with the SCL7A11si-transfected group, co-transfection of SCL7A11si and p53si presented a significantly lower level of ROS, indicating that, potentially, ROS induction by p53 occurred partially by suppressing SCL7A11 (Fig. 3C).

Expression of p53 induced by erastin exposure contributes to the cytotoxic effect on A549 cells, leading to ferroptotic and apoptotic death. To evaluate the effects of p53 induced by erastin exposure on A549 cells, the CFSE/PI double staining or CCK-8 assay was performed to detect the survival rate or cell viability of $\mathrm{A} 549$ cells exposed to the $\mathrm{IC}_{50}$ concentration of erastin for $24 \mathrm{~h}$. As illustrated in Fig. 4A and B, erastin potently inhibited A549 cell survival, as evidenced by CCK-8 optical density (OD) reduction. Knockdown of p53 attenuated erastin-exerted cytotoxicity. According to research, erastin triggers ferroptosis, which is a unique iron-dependent form of non-apoptotic death, prompting us to identify the $\mathrm{p} 53$-contributed cytotoxic effect on A549 cells after erastin exposure. Under the erastin-exposed condition, ferrostatin-1 (Fer-1) or Z-VAD-FMK was employed to inhibit ferroptotic or apoptotic death, respectively, and the role of p53 in inducing cytotoxicity was assessed. After confirming the significant decrease in cell viability following erastin exposure compared with untreated cells (data not shown), Fer-1 or Z-VAD-FMK was applied to reveal the effects of p53 on erastin-induced ferroptotic and apoptotic death. By inhibiting apoptotic death confirmed by detecting caspase-3 activity (Fig. 4C), knockdown of p53 inhibited erastin-induced cell death, as detected by CFSE/PI double staining (Fig. 4D). By inhibiting ferroptotic death without 

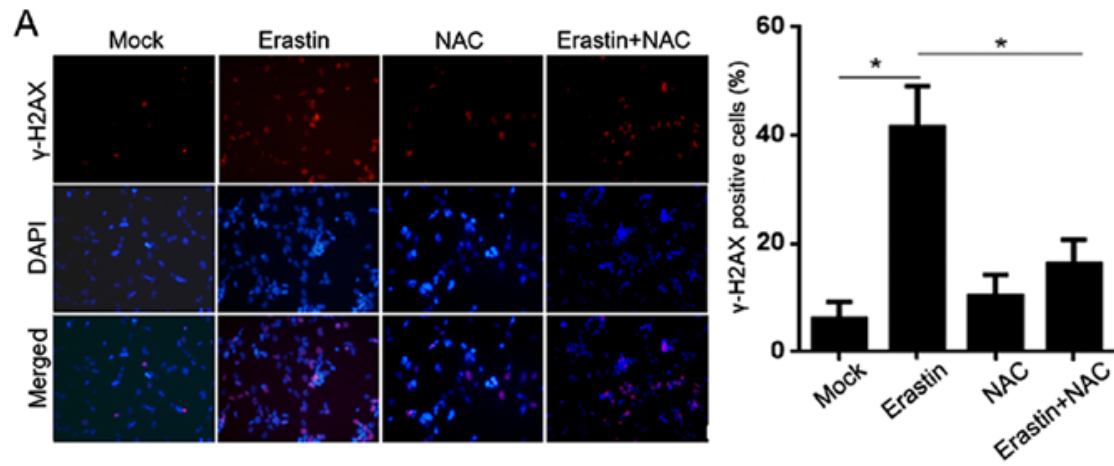

B
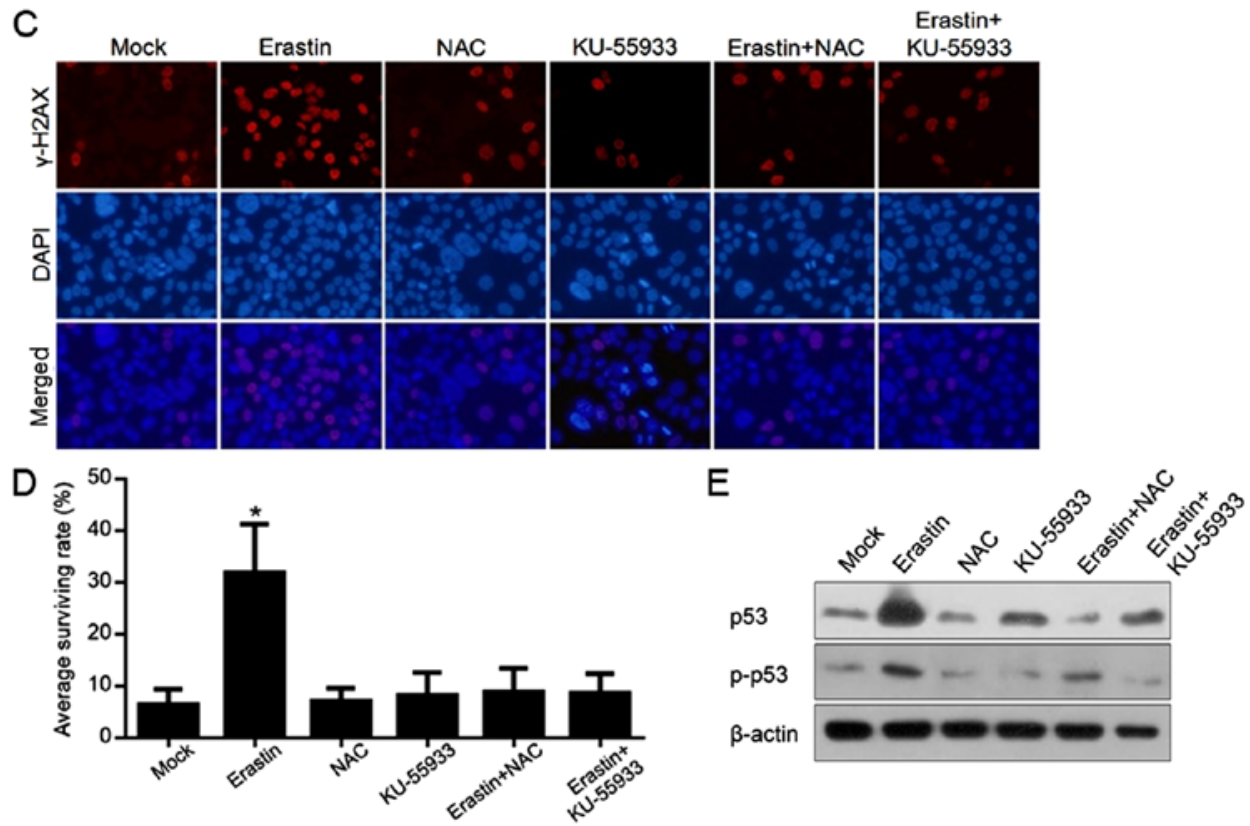

Figure 2. Accumulated ROS activate p53 via inducing the DNA damage response. (A) Formation of $\gamma$-H2AX positive-stained cells after exposure to erastin with or without NAC. The cells were imaged (left panel), and the positive rate was quantified (right panel). $\gamma$-H2AX (red) and nuclei (DAPI) were stained separately. (B) The levels of total and phosphorylated p53 were analysed by western blotting. (C) The effect of KU-55933 on the erastin-induced DNA damage response was detected. Images were acquired at a magnification of x200. $\gamma$-H2AX (red) and nuclei (DAPI) were stained separately. (D) Survival rates were calculated by calculating the $\gamma$-H2AX positive-stained ratio. (E) Detection of total and phosphorylated p53 was performed to determine the necessity of the ROS-induced DNA damage response on p53 activation. "P<0.05 vs. the Mock, NAC, KU-55933, Erastin+NAC and Erastin+KU-55933 group.
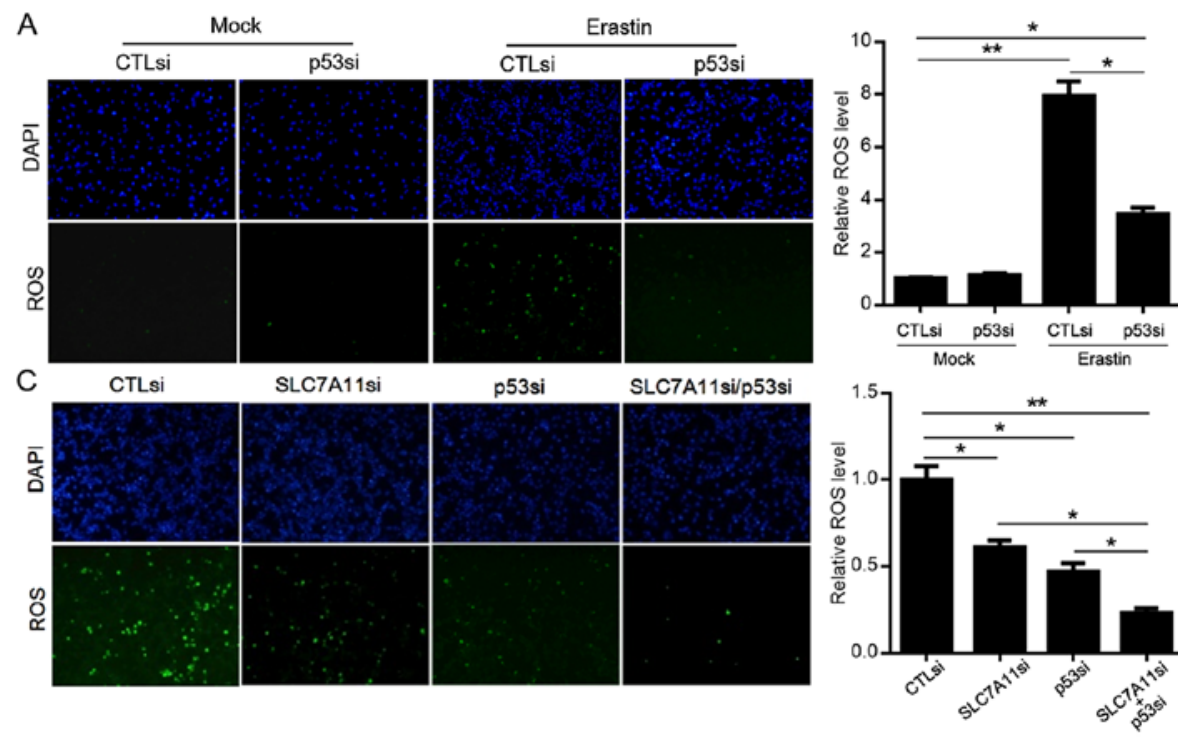

B

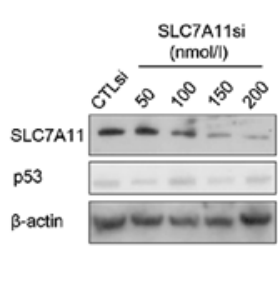

Figure 3. p53 promotes ROS induction by erastin exposure partially dependent on the expression of SLC7A11. (A) ROS generation in CTLsi- or p53si-transfected A549 cells after Mock or erastin exposure was detected by fluorescence microscopy (left panel) and flow cytometry (right panel). ${ }^{*} \mathrm{P}<0.05,{ }^{* *} \mathrm{P}<0.01 \mathrm{vs}$. the CTLsi group. (B) Semi-quantitative western blotting was performed to detect the knockdown efficiency by SLC7A11si in A549 cells. (C) ROS generation in CTLsi-, SLC7A11si-, p53si- or SCL7A11si+p53si-transfected A549 cells after Mock or erastin exposure was detected by fluorescence microscopy (left panel) and flow cytometry (right panel). ${ }^{*} \mathrm{P}<0.05$ vs. the CTLsi, SLC7A11si or p53si group. ${ }^{* *} \mathrm{P}<0.01$ vs. the CTLsi group. 

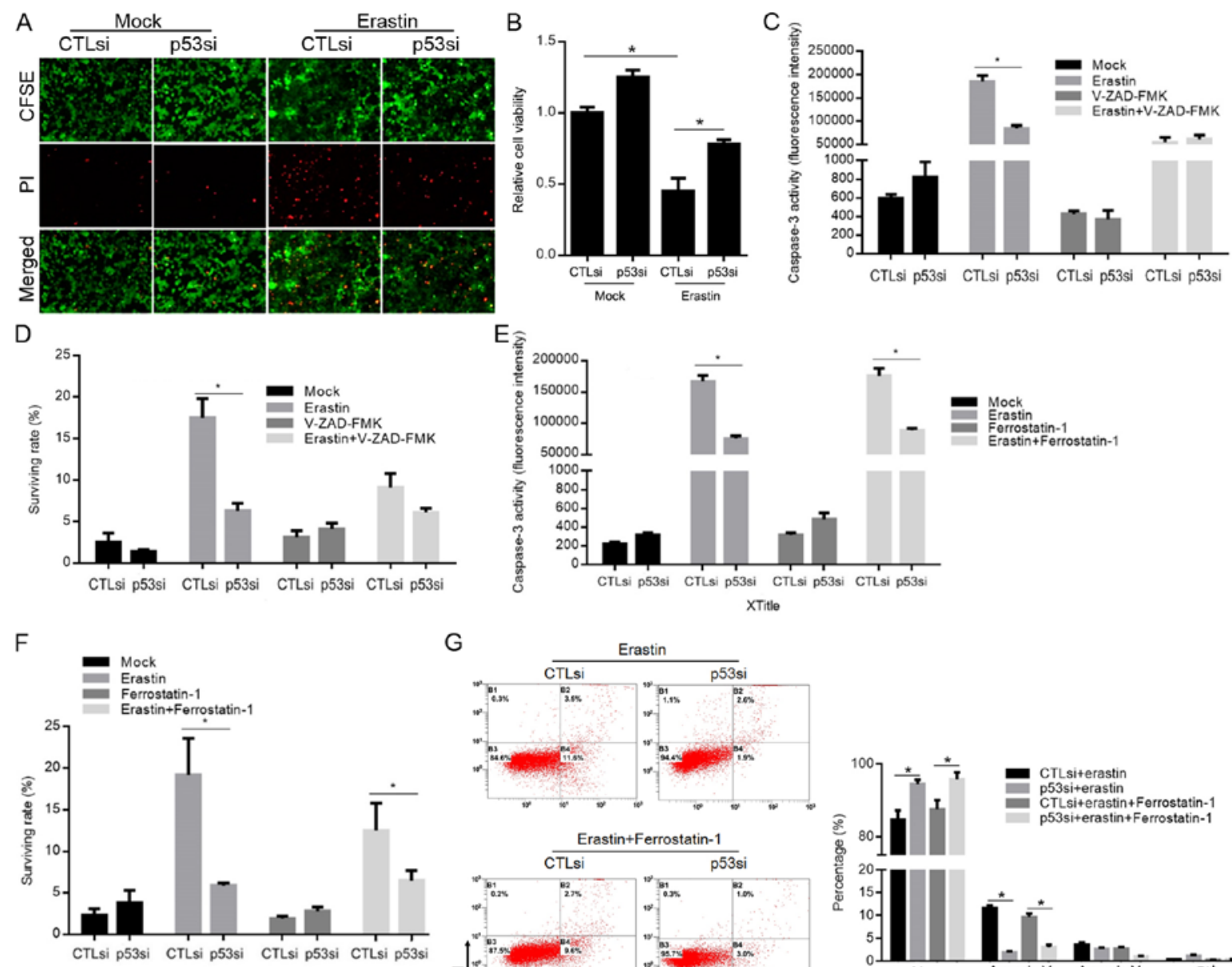

G
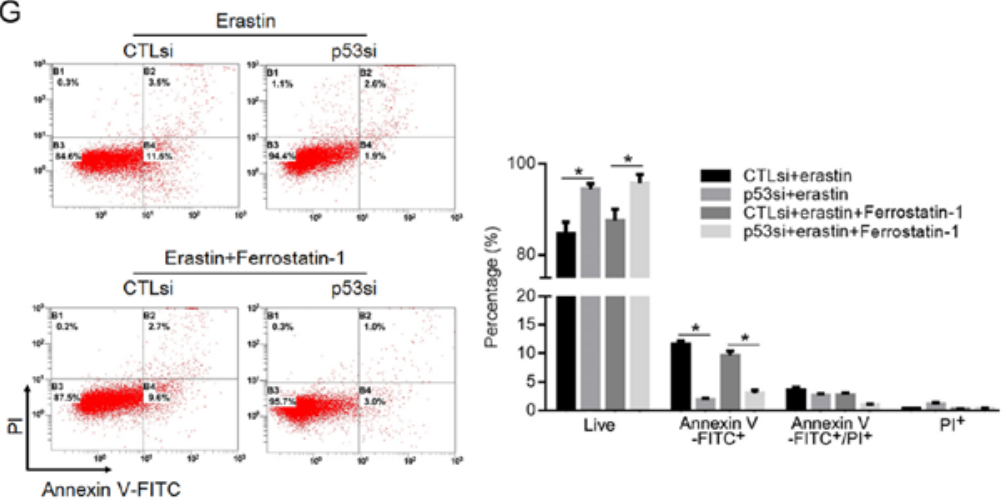

Figure 4. p53 induced by erastin exposure promotes ferroptotic and apoptotic death in A549 cells. (A) CFSE/PI double staining was performed to detect the role of $\mathrm{p} 53$ induced by erastin exposure. (B) CCK-8 assay was performed to assess cell viability with or without erastin treatment. (C) Inhibition of caspase-3 activity confirmed the inhibitory effect of V-ZAD-FMK on apoptosis. (D) After inhibiting apoptotic death, the role of p53 in promoting cell death was detected by CFSE/PI double staining, and the cell survival rate was calculated. (E) Ferrostatin-1 presented no disturbance of caspase-3 activity. (F) After inhibiting ferroptotic death, the role of p53 in promoting cell death was detected by CFSE/PI double staining and (G) Annexin V-FITC/PI double staining. ${ }^{*}<0.05$ vs. the CTLsi or CTL+erastin group.

disturbing caspase-3 activity (Fig. 4E), knockdown of p53 also exerted an inhibitory effect of apoptotic death induced by erastin exposure (Fig. 4F and G).

p53 induced by erastin exposure exerts cytostatic effects on A549 cells. To test the effect of p53 induced by erastin on A549 cell proliferation, A549 cells were treated with the $\mathrm{IC}_{30}$ concentration of erastin $(3.12 \mu \mathrm{M})$ for $24 \mathrm{~h}$. We subjected the treated cells to EdU staining, which is a proliferation marker, and observed that erastin treatment promoted stalled replication, which was greatly rescued by p53 knockdown, as shown by the EdU incorporation (Fig. 5A). This result indicated that erastin treatment inhibited cellular proliferation via the presence of p53. Consistently, analysis of the cell cycle distribution by flow cytometry demonstrated that erastin treatment induced slight accumulation in the $G_{0} / G_{1}$ phase that was reversed by p53 knockdown (Fig. 5B). Since both accumulated ROS and activated p53 mediate the cell accumulation in the $\mathrm{G}_{0} / \mathrm{G}_{1}$ phase (13), we next examined the related kinetics. While activation of $\mathrm{p} 53$ or $\mathrm{G}_{0} / \mathrm{G}_{1}$ phase accumulation was detectable at 12,18 or $24 \mathrm{~h}$ (Fig. 5C), accumulation of ROS was detected at $3 \mathrm{~h}$ after erastin treatment (Fig. 5D). We also determined the effects of erastin-induced ROS on the cell cycle arrest by employing the ROS scavenger $N$-L-acetylcysteine (NAC) with erastin. In Fig. 5E, scavenging of erastin-induced ROS was eliminated by pretreatment of the cells with NAC, leading to entry into the cell cycle. Collectively, erastin exposure generated ROS, thus activating p53, which demonstrated critical cytotoxic and cytostatic effects in A549 cells.

\section{Discussion}

Erastin exposure has been found to exert a cytotoxic effect in numerous cancer cells, including colon (16), lung cancer (17) and leukaemia cells (18), via inducing apoptotic cell death. Recently, it was also revealed that erastin treatment exerts cytotoxic effects via promoting ferroptotic cell death, which is morphologically, biochemically and genetically distinct from apoptosis, necrosis and autophagy (4). Erastin exposure rapidly causes iron-dependent accumulation of lipid ROS, which is a direct trigger of cell death (3). It is also well-known that the accumulation of ROS results in the release of cytochrome $c$ 
A

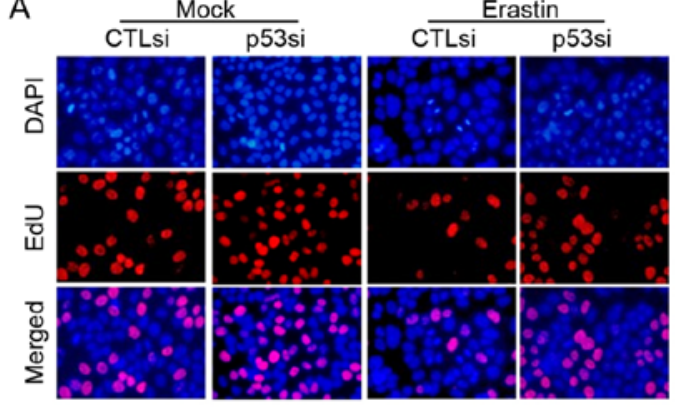

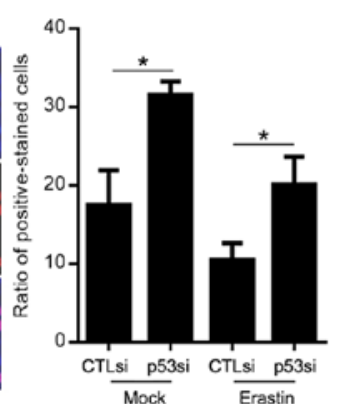

B

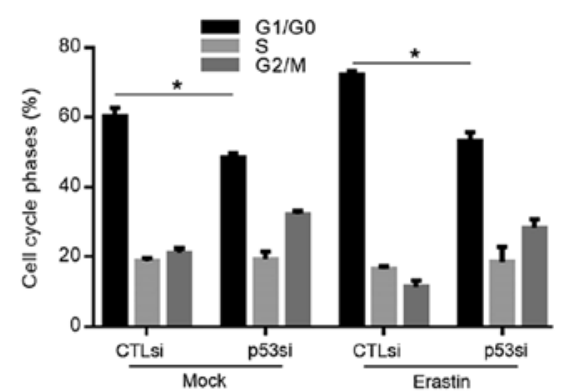

$\mathrm{C}$
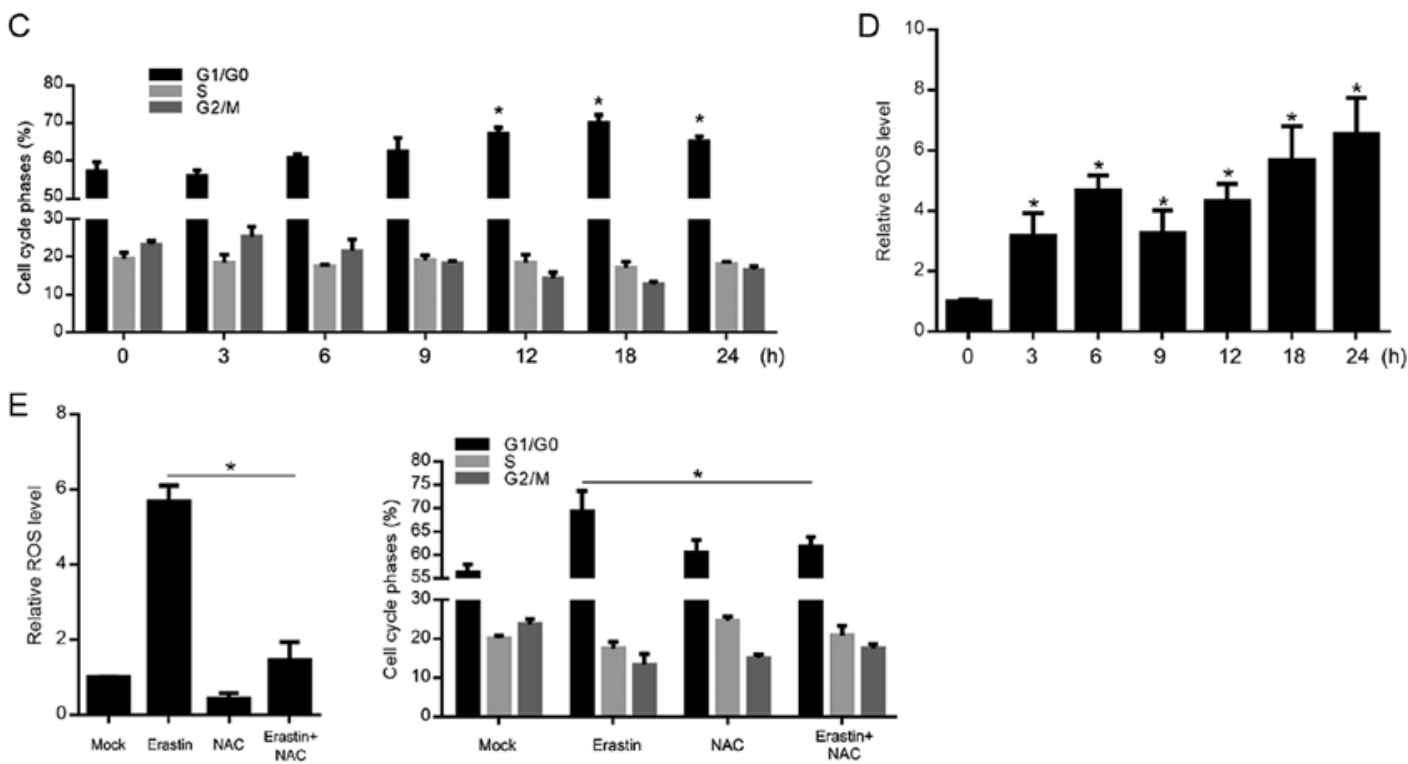

Figure 5. p53 induced by erastin exposure exerts cytostatic effects via blockade of the cell cycle at the G1-phase in A549 cells. (A) EdU staining was performed to detect the effects of p53 on cell proliferation following erastin treatment. (B) The cell cycle distribution was detected by flow cytometry after PI staining. (C) The cell cycle distribution and (D) relative ROS levels over time were detected. (E) After scavenging ROS by NAC pretreatment, the relative ROS levels (left panel) and cell cycle distribution (right panel) were detected. " $\mathrm{P}<0.05$ vs. the CTLsi, p53si or erastin group.

into the cytosol, thus activating caspase cleavage and initiating the apoptotic process $(19,20)$. p53, as the most well-known tumour suppressor, is tightly associated with the regulation of ROS generation and the ROS-induced apoptotic process (21). However, how ROS-induced activated p53 following erastin exposure exerts cytotoxic or cytostatic effects or how induced ROS regulates the activity of p53 remains largely elusive.

In the present study, we demonstrated that erastin-induced ROS regulated and activated p53, as well as partially exerted cytotoxic and cytostatic effects on A549 cells. Following the addition of erastin to A549 cells, we observed an increased ROS level and increased expression of $\mathrm{p} 53$ and phosphorylated p53 over time (Fig. 1A and B). This increase was followed by an increased expression of p53 downstream target genes Bax and p21 (Fig. 1C) and decreased expression of SLC7A11 (Fig. 1D). Since NAC scavenging generated ROS by erastin exposure (22), no detectable change in the p53 expression level and its downstream target genes was observed, indicating that erastin-induced ROS may play a critical role in inducing p53 and subsequently transcriptionally activating its downstream target genes. By considering that a decrease in the SLC7A11 level increased the ROS level (6), we tested whether knockdown of SLC7A11 was accompanied by changes in the ROS level. Notably, we observed that not only SLC7A11 was responsible for the ROS level, but knockdown of p53 also partially decreased the ROS level in an SLC7A11-independent mechanism (Fig. 3). We hypothesised that ROS generation may collaborate with other p53-dependent mechanisms.

Our results demonstrated that erastin exposure led to apoptotic and ferroptotic cell death that was partially dependent on p53 (Fig. 4). We speculated that activated p53 may play a critical role in promoting both apoptotic and ferroptotic cell death, possibly through the activity of its downstream target genes. For example, Bax translocates from the cytosol to the mitochondria upon stress, leading to cytochrome $c$ release and subsequent caspase cascade (23), which is a direct target gene of p53 and was found to be regulated via p53 after erastin exposure (Fig. 1). As expected, both p53 knockdown and inhibition of apoptotic cell death by involving the caspase inhibitor Z-VAD-FMK attenuated the cytotoxic effect of erastin treatment (Fig. 4).

A previous study has shown that activated p53 transcriptionally regulates genes such as p21, 14-3-3o, Reprimo and GADD45 to inhibit cell cycle entry (24). Our results indicated that, following exposure to a comparatively low concentration of erastin, activated p53 may activate one or several pathways that limit cell-cycle progression. This outcome was consistent with the studies of ferritin showing that upregulated ferritin facilitated growth arrest via the induction of cyclin-dependent kinase inhibitor p21 (25). 
In summary, exposure to erastin induced ROS generation and subsequent p53 activation. As a feedback loop, activated p53 partially contributed to induce ROS generation, potentially through post-transcriptional suppression of SLC7A11. In addition, p53 activation contributed to erastin-induced cytostatic effects via arresting the cell cycle at the G1-phase. Collectively, the presence of p53 sensitised lung cancer cells to erastin-induced cytotoxic and cytostatic effects.

\section{Acknowledgements}

We would like to thank Dr Tao Hong (Department of Anesthesiology, Chongqing People's Hospital, Chongqing, China) for his helpful English editing work.

\section{Funding}

The present study was supported by the Science Foundation of Sichuan Provincial Hospital (no. 30305031023).

\section{Availability of data and materials}

The datasets used during the present study are available from the corresponding author upon reasonable request.

\section{Authors' contributions}

$\mathrm{CH}$ and RJ designed part of the experiments. MY was involved in performing cell culture relative experiments. JD and PL performed the gene expressing analysis, cell transfection and treatment experiments. WS was involved in the molecular experiments, data analysis and writing of the manuscript. All authors read and approved the manuscript and agree to be accountable for all aspects of the research in ensuring that the accuracy or integrity of any part of the work are appropriately investigated and resolved.

\section{Ethics approval and consent to participate}

Not applicable.

\section{Patient consent for publication}

Not applicable.

\section{Competing interests}

The authors declare that they have no competing interests.

\section{References}

1. Yagoda N, von Rechenberg M, Zaganjor E, Bauer AJ, Yang WS, Fridman D, Wolpaw AJ, Smukste I, Peltier JM, Boniface JJ, et al RAS-RAF-MEK-dependent oxidative cell death involving voltage-dependent anion channels. Nature 447: 864-868, 2007.

2. Dolma S,Lessnick SL,Hahn WC and Stockwell BR: Identification of genotype-selective antitumor agents using synthetic lethal chemical screening in engineered human tumor cells. Cancer Cell 3: 285-296, 2003

3. Dixon SJ, Lemberg KM, Lamprecht MR, Skouta R, Zaitsev EM, Gleason CE, Patel DN, Bauer AJ, Cantley AM, Yang WS, et al: Ferroptosis: An iron-dependent form of non-apoptotic cell death. Cell 149: 1060-1072, 2012.
4. Huo H, Zhou Z, Qin J, Liu W, Wang B and Gu Y: Erastin Disrupts mitochondrial permeability transition pore (mPTP) and induces apoptotic death of colorectal cancer cells. PLoS One 11: e0154605, 2016.

5. Vogelstein B, Lane D and Levine AJ: Surfing the p53 network. Nature 408: 307-310, 2000.

6. Jiang L, Kon N, Li T, Wang SJ, Su T, Hibshoosh H, Baer R and $\mathrm{Gu}$ W: Ferroptosis as a p53-mediated activity during tumour suppression. Nature 520: 57-62, 2015.

7. Gao M, Monian P, Quadri N, Ramasamy R and Jiang X: Glutaminolysis and transferrin regulate ferroptosis. Mol Cell 59: 298-308, 2015.

8. Tsai MH, Liu JF, Chiang YC, Hu SC, Hsu LF, Lin YC, Lin ZC, Lee HC, Chen MC, Huang CL and Lee CW: Artocarpin, an isoprenyl flavonoid, induces p53-dependent or independent apoptosis via ROS-mediated MAPKs and Akt activation in non-small cell lung cancer cells. Oncotarget 8: 28342-28358, 2017.

9. Zhou Z, Yin Y, Chang Q, Sun G, Lin J and Dai Y: Downregulation of B-myb promotes senescence via the ROS-mediated p53/p21 pathway, in vascular endothelial cells. Cell Prolif 50, 2017.

10. Assaily W, Rubinger DA, Wheaton K, Lin Y, Ma W, Xuan W, Brown-Endres L, Tsuchihara K, Mak TW and Benchimol S: ROS-mediated p53 induction of Lpin1 regulates fatty acid oxidation in response to nutritional stress. Mol Cell 44: 491-501, 2011.

11. Seo SU, Cho HK, Min KJ, Woo SM, Kim S, Park JW, Kim SH, Choi YH, Keum YS, Hyun JW, et al: Thioridazine enhances sensitivity to carboplatin in human head and neck cancer cells through downregulation of c-FLIP and Mcl-1 expression. Cell Death Dis 8: e2599, 2017.

12. Wang SJ, Li D, Ou Y, Jiang L, Chen Y, Zhao Y and Gu W: Acetylation is crucial for p53-mediated ferroptosis and tumor suppression. Cell Rep 17: 366-373, 2016.

13. Shi Y, Nikulenkov F, Zawacka-Pankau J, Li H, Gabdoulline R, $\mathrm{Xu}$ J, Eriksson S, Hedström E, Issaeva N, Kel A, et al: ROS-dependent activation of JNK converts p53 into an efficient inhibitor of oncogenes leading to robust apoptosis. Cell Death Differ 21: 612-623, 2014.

14. Yang J, Zhao X, Tang M, Li L, Lei Y, Cheng P, Guo W, Zheng Y, Wang $\mathrm{W}$, Luo $\mathrm{N}$, et al: The role of ROS and subsequent DNA-damage response in PUMA-induced apoptosis of ovarian cancer cells. Oncotarget 8: 23492-23506, 2017.

15. An JJ, Shi KJ, Wei W, Hua FY, Ci YL, Jiang Q, Li F, Wu P, Hui KY, Yang Y and Xu CM: The ROS/JNK/ATF2 pathway mediates selenite-induced leukemia NB4 cell cycle arrest and apoptosis in vitro and in vivo. Cell Death Dis 4: e973, 2013.

16. Suh DH, Kim MK, Kim HS, Chung HH and Song YS: Mitochondrial permeability transition pore as a selective target for anti-cancer therapy. Front Oncol 3: 41, 2013.

17. Yamaguchi H, Hsu JL, Chen CT, Wang YN, Hsu MC, Chang SS, Du Y, Ko HW, Herbst R and Hung MC: Caspase-independent cell death is involved in the negative effect of EGFR inhibitors on cisplatin in non-small cell lung cancer cells. Clin Cancer Res 19: 845-854, 2013

18. Aresvik DM, Pettersen RD, Abrahamsen TG and Wright MS: 5-Fluorouracil-induced death of Jurkat T-cells-A role for caspases and MCL-1. Anticancer Res 30: 3879-3887, 2010.

19. Siskind LJ: Mitochondrial ceramide and the induction of apoptosis. J Bioenerg Biomembr 37: 143-153, 2005.

20. Colombini M: Ceramide channels and their role in mitochondria-mediated apoptosis. Biochim Biophys Acta 1797: 1239-1244, 2010.

21. Lago CU, Sung HJ, Ma W, Wang PY and Hwang PM: p53, Aerobic metabolism, and cancer. Antioxid Redox Signal 15: 1739-1748, 2011.

22. Downs I, Liu J, Aw TY, Adegboyega PA and Ajuebor MN: The ROS scavenger, NAC, regulates hepatic Va14iNKT cells signaling during Fas $\mathrm{mAb}$-dependent fulminant liver failure. PLoS One 7: e38051, 2012.

23. Jürgensmeier JM, Xie Z, Deveraux Q, Ellerby L, Bredesen D and Reed JC: Bax directly induces release of cytochrome $c$ from isolated mitochondria. Proc Natl Acad Sci USA 95: 4997-5002, 1998.

24. Horn HF and Vousden KH: Coping with stress: Multiple ways to activate p53. Oncogene 26: 1306-1316, 2007.

25. Zhang F, Wang W, Tsuji Y, Torti SV and Torti FM: Post-transcriptional modulation of iron homeostasis during p53-dependent growth arrest. J Biol Chem 283: 33911-33918, 2008. 